\title{
The dark energy survey and operations: years 1 to 3
}

\section{H. T. Diehl, E. Neilsen, R. Gruendl, B. Yanny, T. M. C. Abbott, et al.}

H. T. Diehl, E. Neilsen, R. Gruendl, B. Yanny, T. M. C. Abbott, J. Aleksić, S. Allam, J. Annis, E. Balbinot, M. Baumer, L. Beaufore, K. Bechtol, G. Bernstein, S. Birrer, C. Bonnett, D. Brout, C. Bruderer, E. J. Buckley-Geer, D. Capozzi, A. Carnero Rosell, F. J. Castander, R. Cawthon, C. Chang, L. Clerkin, R. Covarrubias, C. Cuhna, C. D'Andrea, L. da Costa, R. Das, C. Davis, J. Dietrich, A. Drlica-Wagner, A. Elliott, T. F. Eifler, J. Etherington, B. L. Flaugher, J. Frieman, A. Fausti Neto, M. G. Fernández, C. Furlanetto, D. Gangkofner, D. W. Gerdes, D. A. Goldstein, K. Grabowski, R. R. Gupta, S. Hamilton, H. Head, J. Helsby, D. Hollowood, K. Honscheid, D. James, M. Johnson, M. W. G. Johnson, S. Jouvel, T. Kacprzac, S. Kent, R. Kessler, A. Kim, E. Krause, C. I. Krawiec, A. Kremin, R. Kron, S. Kuhlmann, N. Kuropatkin, O. Lahav, J. Lasker, T. S. Li, E. Luque, N. Maccrann, M. March, J. Marshall, N. P. Mondrik, E. P. Morganson, D. Mudd, A. Nadolski, P. Nugent, P. Melchior, F. Menanteau, D. Q. Nagasawa, B. Nord, R. Ogando, L. Old, A. Palmese, D. Petravick, A. A. Plazas, A. Pujol, A. B. A. Queiroz, K. Reil, A. K. Romer, R. Rosenfeld, A. Roodman, P. Rooney, M. Sako, A. I. Salvador, C. Sánchez, E. Sánchez Álvaro, B. X. Santiago, A. Schooneveld, M. Schubnell, E.

Sheldon, A. Smith, R. C. Smith, M. Soares-Santos, F. Sobreira, M. Soumagnac, H. Spinka, S. S. Tie, D. Tucker, V. Vikram, K. Vivas, A. R. Walker, W. Wester, M. Wiesner, H. Wilcox, P. Williams, A.

Zenteno, Y. Zhang, Z. Zhang, "The dark energy survey and operations: years 1 to 3," Proc. SPIE 9910, Observatory Operations: Strategies, Processes, and Systems VI, 99101D (15 July 2016); doi:

10.1117/12.2233157

Event: SPIE Astronomical Telescopes + Instrumentation, 2016, Edinburgh, United Kingdom 


\section{The Dark Energy Survey and Operations: Years 1 to 3}

H. T. Diehl ${ }^{\text {la }}$, E. Neilsen ${ }^{\mathrm{a}}$, R. Gruendl, ${ }^{\mathrm{b}, \mathrm{c}}$, B. Yanny ${ }^{\mathrm{a}}$, T. M. C. Abbott ${ }^{\mathrm{d}}$, J. Aleksiće ${ }^{\mathrm{e}}$, S. Allam ${ }^{\mathrm{a}}$, J. Annis $^{\mathrm{a}}$, E. Balbinot ${ }^{\mathrm{f}}$, M. Baumer ${ }^{\mathrm{g}}$, L. Beaufore ${ }^{\mathrm{h}}, \mathrm{K}$. Bechtol ${ }^{\mathrm{i}, \mathrm{j}}$, G. Bernstein ${ }^{\mathrm{k}}$, S. Birrer ${ }^{\mathrm{l}}$, C. Bonnett $^{\mathrm{e}}$, D. Brout ${ }^{\mathrm{k}}$, C. Bruderer ${ }^{1}$, E. J. Buckley-Geer ${ }^{\mathrm{a}}$, D. Capozzi ${ }^{\mathrm{m}}$, A. Carnero Rosell ${ }^{\mathrm{m}, \mathrm{g}}$, F. J. Castander $^{\mathrm{n}}$, R. Cawthon ${ }^{\mathrm{i}}$, C. Chang, L. Clerkin ${ }^{\mathrm{o}}$, R. Covarrubias ${ }^{\mathrm{b}}$, C. Cuhna ${ }^{\mathrm{p}}$, C. D'Andrea ${ }^{\mathrm{m}, \mathrm{q}}, \mathrm{L}$. da Costa $^{r}$, R. Das ${ }^{\mathrm{s}}$, C. Davis ${ }^{\mathrm{p}}$, J. Dietrich ${ }^{\mathrm{t}, \mathrm{u}}$, A. Drlica-Wagner ${ }^{\mathrm{a}}$, A. Elliott ${ }^{\mathrm{h}}$, T. F. Eifler ${ }^{\mathrm{k}, \mathrm{v}}$, J.

Etherington $^{\mathrm{m}}$, B. L. Flaugher, J. Frieman ${ }^{\mathrm{a}}$, A. Fausti Neto ${ }^{\mathrm{r}}$, M. G. Fernández ${ }^{\mathrm{n}}$, C. Furlanetto ${ }^{\mathrm{w}}$, D. Gangkofner, ${ }^{\text {t,u }}$, D. W. Gerdes ${ }^{\mathrm{s}}$, D. A. Goldstein ${ }^{\mathrm{x}, \mathrm{y}}$, K. Grabowski ${ }^{\mathrm{a}}$, R. R. Gupta ${ }^{\mathrm{z}}$, S. Hamilton ${ }^{\mathrm{s}}, \mathrm{H}$. Head $^{\text {aa }}$, J. Helsby ${ }^{\mathrm{i}}$, D. Hollowood ${ }^{\text {ab }}$, K. Honscheid ${ }^{\text {h }}$, D. James ${ }^{\mathrm{d}}$, M. Johnson ${ }^{\mathrm{b}}$, M. W. G. Johnson ${ }^{\mathrm{b}}$, S. Jouvel ${ }^{\mathrm{o}}$, T. Kacprzac ${ }^{\mathrm{o}}$, S. Kent ${ }^{\mathrm{a}}$, R. Kessler, A. Kim ${ }^{\mathrm{x}}$, E. Krause, ${ }^{\mathrm{k}, \mathrm{i}}$, C. I. Krawiec ${ }^{\mathrm{k}}$, A. Kremin's, R. Kron ${ }^{\text {a }}$, S. Kuhlmann , N. Kuropatkin ${ }^{\text {a }}$, O. Lahav ${ }^{\circ}$, J. Lasker ${ }^{\mathrm{i}}$, T. S. Li ${ }^{\text {ac }}$, E. Luque ${ }^{\text {ad, }}$, N. Maccrann $^{\text {ae }}$, M. March ${ }^{\mathrm{k}}$, J. Marshall $^{\text {ac }}$, N. P. Mondrik ${ }^{\text {ac }}$, E. P. Morganson ${ }^{\mathrm{b}, \mathrm{c}}$, D. Mudd ${ }^{\mathrm{af}}$, A. Nadolski $^{\mathrm{c}}$, P. Nugent ${ }^{\mathrm{x}}$, P. Melchior ${ }^{\mathrm{u}, \mathrm{ag}}$, F. Menanteau ${ }^{\mathrm{b}, \mathrm{c}}$, D. Q. Nagasawa ${ }^{\mathrm{ac}}, \mathrm{B}$. Nord ${ }^{\mathrm{a}}$, R.

Ogando $^{\mathrm{r}, \text { ah }}$, L. Old ${ }^{\mathrm{w}}$, A. Palmese ${ }^{\mathrm{o}}$, D. Petravick ${ }^{\mathrm{b}}$, A. A. Plazas ${ }^{\mathrm{ai,v}}$, A. Pujol ${ }^{\mathrm{n}}$, A. B. A. Queiroz ${ }^{\text {ad, }}$, K. Reil ${ }^{\text {paj }}$, A. K. Romer ${ }^{\text {ak }}$, R. Rosenfeld ${ }^{\text {ak }}$, A. Roodman ${ }^{\text {paj }}$, P. Rooney ${ }^{\text {ak }}$, M. Sako ${ }^{\mathrm{k}}$, A. I. Salvador $^{\mathrm{am}}$, C. Sánchez ${ }^{\mathrm{e}}$, E. Sánchez Álvaro ${ }^{\text {an }}$, B. X. Santiago ${ }^{\text {ad,k }}$, A. Schooneveld ${ }^{\mathrm{ao}}$, M. Schubnell $^{\mathrm{s}}$, E. Sheldon ${ }^{\mathrm{ai}}$, A. Smith ${ }^{\mathrm{aa}}$, R. C. Smith ${ }^{\mathrm{d}}$, M. Soares-Santos ${ }^{\mathrm{a}}$, F. Sobreira, ${ }^{\mathrm{a}, \mathrm{al}}$, M.

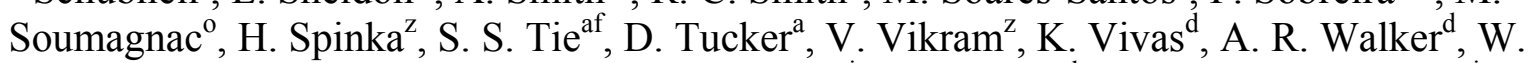
Wester $^{\mathrm{a}}$, M. Wiesner ${ }^{\mathrm{a}}$, H. Wilcox ${ }^{\mathrm{m}}$, P. Williams, ${ }^{\mathrm{i}}$, . Zenteno ${ }^{\mathrm{d}}$, Y. Zhang ${ }^{\mathrm{a}, \mathrm{s}}$, Z. Zhang ${ }^{\text {aj }}$

${ }^{a}$ Fermi National Accelerator Laboratory, P.O. Box 500, Batavia, IL 60510, USA; ${ }^{b}$ National Center for Supercomputing Applications, 1205 West Clark St., Urbana, IL 61801, USA; ${ }^{c}$ Department of Astronomy, University of Illinois at Urbana-Champaign, W. Green Street, Urbana, IL 61801, USA; ${ }^{\mathrm{d}}$ National Optical Astronomy Observatory, Cerro Tololo InterAmerican Observatory, Casilla 603, La Serena, Chile; ${ }^{\mathrm{e}}$ Institut de Física Altes Energies (IFAE), The Barcelona Institute of Science and Technology, Campus UAB, 08193, Bellaterra

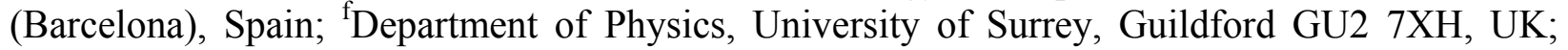
${ }^{g}$ Department of Physics, Stanford University, 382 Via Pueblo Mall, Stanford, CA 94305, USA; ${ }^{\mathrm{h}}$ Department of Physics, The Ohio State University, Columbus, OH 43210, USA; ${ }^{\mathrm{i}}$ Kavli Institute for Cosmological Physics, University of Chicago, Chicago, IL 60637, USA; ${ }^{j}$ Department of Physics, University of Wisconsin, Madison, WI 53706, USA; ${ }^{\mathrm{k}}$ Department of Physics and Astronomy, University of Pennsylvania, Philadelphia, PA 19104, USA; Institute for Astronomy, Department of Physics, ETH Zurich, Wolfgang-Pauli-Strasse 27, CH-8093 Zurich, Switzerland; ${ }^{\mathrm{m}}$ Institute of Cosmology and Gravitation, University of Portsmouth, Portsmouth, PO1 3FX, UK; ${ }^{\mathrm{n} I n s t i t u t ~ d e ~ C i e ́ n c i e s ~ d e ~ l ' E s p a i ~(I C E, ~ I E E C / C S I C), ~ E-08193 ~ B e l l a t e r r a ~(B a r c e l o n a), ~ S p a i n ; ~}$ ${ }^{\circ}$ Department of Physics and Astronomy, University College London, Gower St., London, WC1E 6BT, UK; ${ }^{\mathrm{p} K a v l i ~ I n s t i t u t e ~ f o r ~ P a r t i c l e ~ A s t r o p h y s i c s ~ a n d ~ C o s m o l o g y, ~} 452$ Lomita Mall, Stanford University, Stanford, CA 94305, USA; ${ }^{\mathrm{q}}$ Department of Physics and Astronomy, University of Southampton, Southampton, SO17 1BJ, UK; ${ }^{\mathrm{r}}$ Laboratório Interinstitucional de e-Astronomia LIneA, Rua Gal. José Cristino 77, Rio de Janeiro, RJ - 20921-400, Brazil; ${ }^{\text {s}}$ Department of Physics, University of Michigan, Ann Arbor, MI 48109, USA; 'University Observatory Munich, Scheinerstrasse 1, 81679 Munich, Germany; "Excellence Cluster Universe, 85748 Garching b.

${ }^{1}$ Diehl@FNAL.GOV; phone 1-630-840-8307.

Observatory Operations: Strategies, Processes, and Systems VI, edited by Alison B. Peck, Robert L. Seaman, Chris R. Benn, Proc. of SPIE Vol. 9910, 99101D - @ 2016 SPIE · CCC code: 0277-786X/16/\$18 · doi: 10.1117/12.2233157 
Munich, Germany; ${ }^{\vee} J e t$ Propulsion Laboratory, California Institute of Technology, 4800 Oak

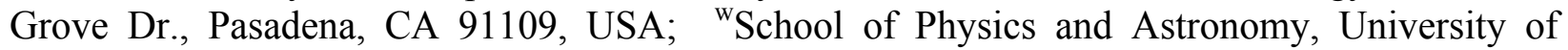
Nottingham, University Park, Nottingham, NG7 2RD, UK; ${ }^{\mathrm{x}}$ Lawrence Berkeley National Laboratory, 1 Cyclotron Road, Berkeley, CA 94720, USA; ${ }^{\mathrm{y}}$ Department of Astronomy, University of California, Berkeley, 501 Campbell Hall, Berkeley, CA 94720, USA; ${ }^{\mathrm{z} A r g o n n e}$

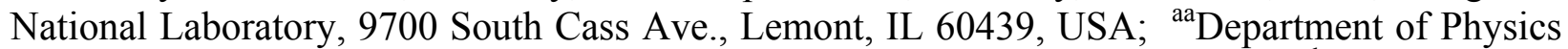
and Astronomy, Austin Peay State University, Clarksville, TN 37044, USA; ${ }^{\text {ab }}$ Department of Physics and Santa Cruz Institute for Particle Physics, University of California, Santa Cruz, CA 95064, USA; ${ }^{\text {ac }}$ George P. and Cynthia Woods Mitchell Institute for Fundamental Physics and Astronomy, and Department of Physics and Astronomy, Texas A\&M University, College

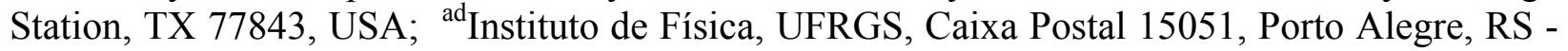

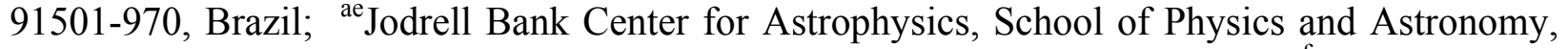

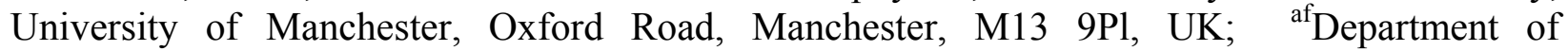
Astronomy, The Ohio State University, Columbus, OH 43210, USA; ${ }^{\mathrm{ag}}$ Center for Cosmology and Astro-Particle Physics, The Ohio State University, Columbus, OH 43210, USA; ah Observatório Nacional, R. Gal. José Cristino 77, BR Rio de Janeiro, RJ 20921-400, Brazil; ${ }^{a i}$ Brookhaven National Laboratory, Building 510, Upton, NY, 11973 USA; ${ }^{\text {aj }}$ SLAC National Accelerator Laboratory, Menlo Park, CA 94025, USA; ${ }^{\mathrm{ak}}$ Department of Physics and Astronomy, Univ. of Sussex, UK; ${ }^{\text {al }}$ Instituto de Física Teórica, Universidade Estadual Paulista, Rua Dr. Bento T. Ferraz 271, São Paulo, SP 01140-070, Brazil; ${ }^{\mathrm{am}}$ Instituto de Física Teórica, UAM-CSIC, Madrid, Spain; ${ }^{\text {an }}$ Centro de Investigaciones Energéticas, Medioambientales y

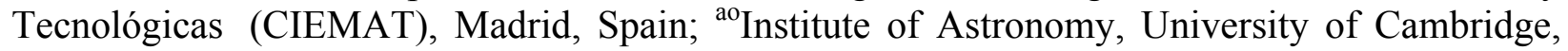
Madingley Road, Cambridge CB3 OHA, UK

\begin{abstract}
The Dark Energy Survey (DES) is an operating optical survey aimed at understanding the accelerating expansion of the universe using four complementary methods: weak gravitational lensing, galaxy cluster counts, baryon acoustic oscillations, and Type Ia supernovae. To perform the 5000 sq-degree wide field and 30 sq-degree supernova surveys, the DES Collaboration built the Dark Energy Camera (DECam), a 3 square-degree, 570-Megapixel CCD camera that was installed at the prime focus of the Blanco 4-meter telescope at the Cerro Tololo Inter-American Observatory (CTIO). DES has completed its third observing season out of a nominal five. This paper describes DES "Year 1" (Y1) to "Year 3" (Y3), the strategy, an outline of the survey operations procedures, the efficiency of operations and the causes of lost observing time. It provides details about the quality of the first three season's data, and describes how we are adjusting the survey strategy in the face of the El Niño Southern Oscillation.
\end{abstract}

Keywords: Cosmology, Dark Energy Survey, Dark Energy Camera, Operations, CTIO

\title{
1. INTRODUCTION
}

The Dark Energy Survey (DES) is an international collaboration, with over 500 scientists from 28 institutions and consortiums in the US, Chile, the UK, Spain, Brazil, Switzerland, and Germany. The wide-field (WF) survey will produce images of 5000 square degrees of the southern galactic cap collected during 525 nights of observing from 2013 to 2018. The WF survey will be accomplished in 10 dither patterns (tilings). Additionally, ten 3-square-degree fields will be imaged repeatedly to produce a supernovae survey The DES [1-2] will measure dark energy parameters using four complementary techniques: galaxy cluster counting, baryon acoustic oscillations, weak gravitational lensing, and Type Ia supernovae. In order to carry out these surveys, the DES Collaboration constructed a new instrument, the Dark Energy Camera. 


\section{The Dark Energy Camera and Auxiliary Detectors}

The Dark Energy Camera (DECam) [3] was designed and built from 2004 to 2011. It consists of a wide-field corrector, a mosaic CCD imager and associated mechanical, optical, and electronic components. The optical corrector has 5 fused-silica optical elements to attain an f/2.7, 2-degree-wide image at the focal plane. DES uses 5 filters, DES g, DES r, DES i, DES z, and DES Y-band, with central wavelengths 473, 642, 784, 926, and $1009 \mathrm{~nm}$, respectively. The focal plane itself has a $42 \mathrm{~cm}$ radius and is populated with $622048 \times 4096$ pixel $250 \mu \mathrm{m}$ thick, fully-depleted, red-sensitive CCDs, for imaging. DECam was delivered to CTIO in a series of shipments starting in early 2010. The imager was the last major component to be shipped, arriving in December 2011. Installation [4-6] followed. "Official First Light" was achieved on September 12, 2012 [7]. DECam was commissioned during September and October 2012. A survey and instrument testing period called "Science Verification" was carried out by the DES Collaboration and "Community Astronomers" during November. Regular "Community Observing" began on December 1, 2012. Science Verification was extended into February 2013, principally to iron out remaining issues with the telescope. As a result, the first observing season for DES was postponed to August 2013. Figure 1 shows a photo of the Dark Energy Camera mounted at the prime focus of the Blanco telescope.

Three auxiliary detectors on the CTIO summit, supplied by DES, provide information for photometric calibration. An All-Sky Radiometric Camera (RASICAM) [3,8-9] is used to monitor the sky using the wavelength range $970 \mathrm{~nm}$ $<\lambda<1250 \mathrm{~nm}$. In this wavelength range, relatively warm clouds are easily distinguished from cold, clear skies. "GPSMon" [3,10] provides a cross-check of the amount of precipitable water vapor (PWV) in the atmosphere. The Atmospheric Transmission Monitoring Camera (aTmCam) [3,11-12] was tested in Y1 and Y2 and operated consistently starting at the beginning of Y2. The aTmCam consists of a Paramount telescope mount and four small telescopes, each with a different narrowband filter, which monitors the brightness of suitable standard stars, thus providing the atmospheric transmission in wavelength regions dominated by the PWV and aerosol optical depth.

\section{The Dark Energy Survey Fields}

DES carries out two interleaved surveys: a 5000 square-degree "wide field" (WF) survey in the southern Galactic Cap and a 30 square-degree time domain survey. The 5000 square-degree "wide-field" (WF) has three main regions (see Figure 2). There is a broad roughly circular region from RA of roughly 0 to 120 degrees and DEC -70 to -10 degrees that provides a large contiguous area for the large-scale structure measurements. There is a wide roughly box-shaped region around the South Pole Telescope (SPT) observing area [13]. Finally, the survey encompasses a part of SDSS Stripe 82 [14], primarily for calibration purposes.

The footprint of a single DECam exposure is roughly hexagonal, constrained to an orientation aligned with celestial coordinates by the equatorial mount of the Blanco telescope; therefore, an area of the sky covering a particular range of R.A. can be covered with minimal gaps and overlaps by using an hexagonal tiling pattern aligned in declination. A "tiling" is a set of exposures, one in each of 5 filters, at pointings arranged in such a pattern. Over wider ranges of R.A. the planar approximation of the sky breaks down, so the strictly hexagonal layout is "broken" every 30 degrees of R.A., resulting in extra overlaps between exposures within a tiling at 30 degree intervals. A single tiling collects useful science data on roughly $83 \%$ of the footprint area due to these breaks, deviation from the planar approximation with each 30 degree R.A. neighborhood, and incomplete coverage within each hex (due to, for example, gaps between CCDs, bad CCDs, and problematic area near the edges of the CCDs). The plan for the DES wide survey specifies 10 separate tilings, each offset from the others by a significant fraction of the camera field of view, such that observations of individual astronomical sources are spread across the focal plane. The $\mathrm{g}, \mathrm{r}, \mathrm{i}$, and $\mathrm{z}$ band exposures are 90 seconds duration. The Y-band exposures are 45 seconds duration through Y3. In Y4 we plan to change the Y-band observations to 90 seconds. See Section 5. 


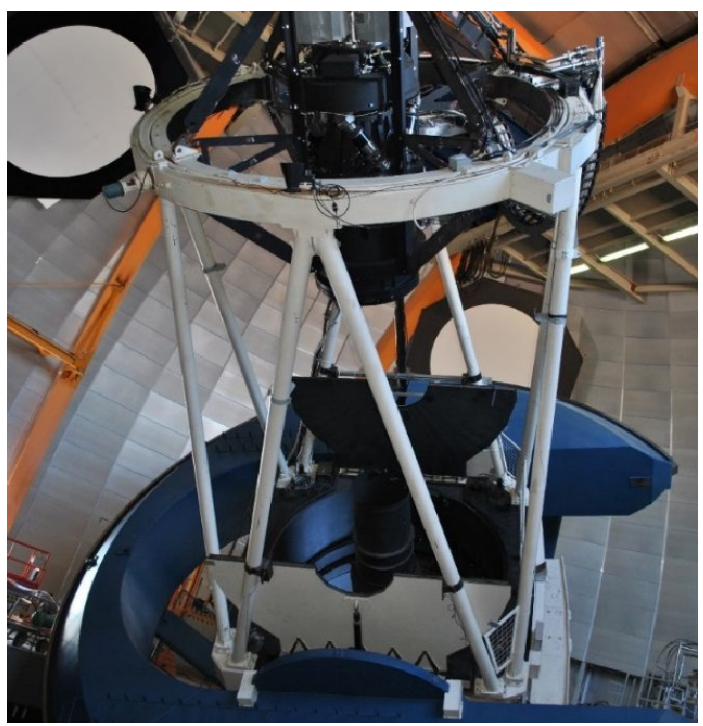

Figure 1 The Dark Energy Camera is mounted at the prime focus of the Blanco $4 \mathrm{~m}$ telescope at CTIO. The covers for the primary mirror are open. The camera assembly, including the support cage, is approximately $3.6 \mathrm{~m}$ long and is secured to the inner telescope ring. The camera, not including the support cage and counterweights, weighs approximately $4350 \mathrm{~kg}$. Subsequent to the date of this photo the cage was retrofitted with aluminum side-covers. The aluminum covers of two of the four readout electronics crates are visible near the top of the camera, just underneath the "Top Cap".

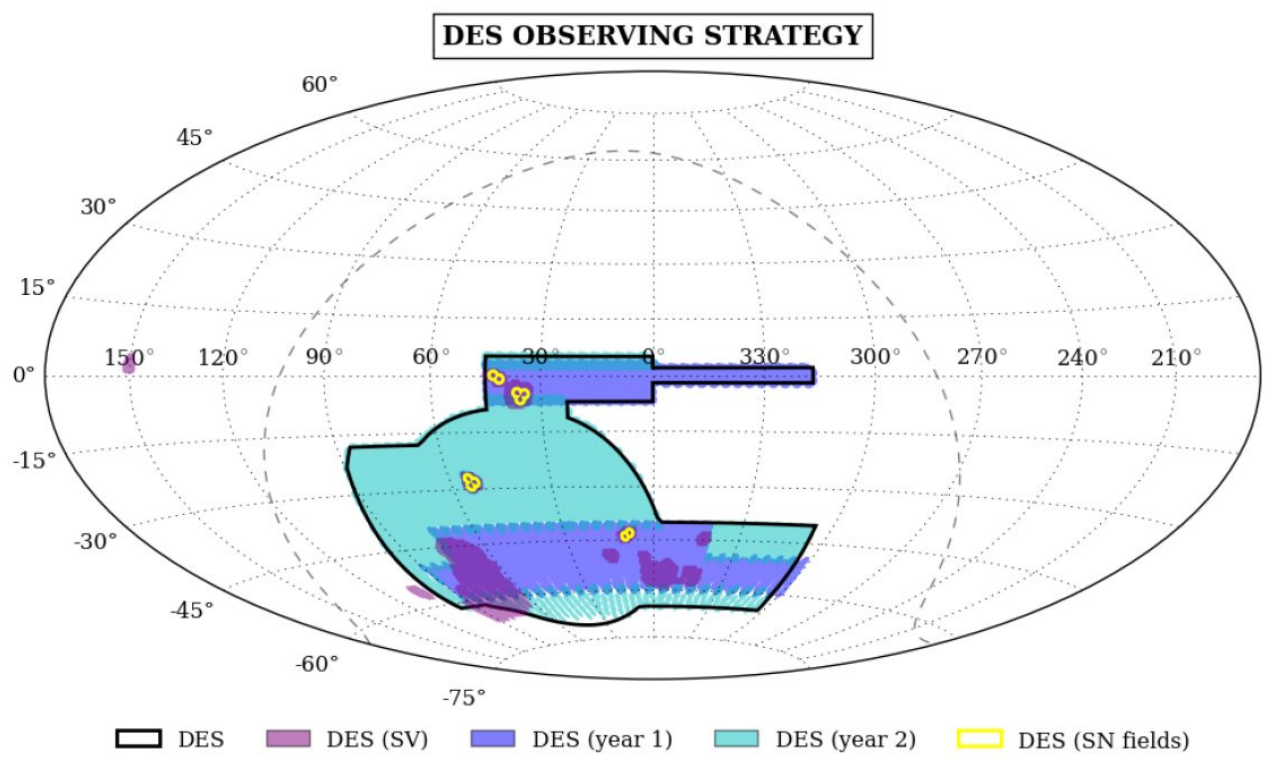

Figure 2. The Dark Energy Survey observing “wide fields" are shown outlined in black on this plot of RA and DEC. During Y1 DES planned to observe the areas outlined in dark blue, which encompass SDSS Stripe 82 (upper) and the SPT area (lower). The legend also shows the color for the SV (purple blotches), SN (yellow), and nominal Y2 areas.

The purpose of the time domain survey is to identify Type Ia SNe through difference imaging [15] and measurement of the light curves. The 10 time domain fields, shown in Table 1, are observed on a regular cadence. The 8 "shallow fields" are observed for single exposures in g-band (175s), r-band (150s), and i-band (200s) and for two images in zband (200s each). The 5 exposures of a shallow field are considered a "sequence" and the sequence is observed consecutively. The two "deep fields" are observed for 3 exposures of 200s each in g-band, for 400s each in r-band, for 5 exposures of 360s each in i-band, and for 11 exposures of 330s each in z-band. The exposures in each given filter for the deep fields are considered a sequence. The exposures for each filter are sequential but the different 
filters might be observed at different times during a single night or on different nights. The limiting magnitudes [15] for difference imaging detections is about 23.5 in each band of the shallow field epochs and about 24.5 for the deep field epochs. Because the telescope pointing is accurate to 5 to 7 arcseconds in both RA and DEC, each SN sequence is preceded by a 10 s exposure that is processed to find the pointing offset correction applied before the first exposure in the sequence starts.

Table 1 RA and DEC (J2000) of the 10 DES supernova fields. Fields C3 and X3 are "deep fields". The other 8 fields are "shallow fields".

\begin{tabular}{|c|c|c|}
\hline $\begin{array}{c}\text { Field } \\
\text { Name }\end{array}$ & RA & DEC \\
\hline E1 & $7.8744(00: 31: 29.9)$ & $-43.0096(-43: 00: 34.6)$ \\
\hline E2 & $9.5000(00: 38: 00.0)$ & $-43.9980(-43: 59: 52.8)$ \\
\hline S1 & $42.8200(02: 51: 16.8)$ & $0.0000(00: 00: 00.0)$ \\
\hline S2 & $41.1944(02: 44: 46.7)$ & $-0.9884(-00: 59: 18.2)$ \\
\hline C1 & $54.2743(03: 37: 05.8)$ & $-27.1116(-27: 06: 41.8)$ \\
\hline C2 & $54.2743(03: 37: 05.8)$ & $-29.0884(-29: 05: 18.2)$ \\
\hline C3 & $52.6484(03: 30: 35.6)$ & $-28.1000(-28: 06: 00.0)$ \\
\hline X1 & $34.4757(02: 17: 54.2)$ & $-4.9295(-04: 55: 46.2)$ \\
\hline X2 & $35.6645(02: 22: 39.5)$ & $-6.4121(-06: 24: 43.6)$ \\
\hline X3 & $36.4500(02: 25: 48.0)$ & $-4.6000(-04: 36: 00.0)$ \\
\hline
\end{tabular}

This paper describes DES "Year 1" (Y1) to "Year 3" (Y3) operations. Section 2 describes the survey procedures, Section 3, the maintenance and improvements to the camera and observing systems. Section 4, the Y1 to Y3 narrative including a description of the strategy and goals for the first three year's data, the efficiency of survey operations and the progress towards the survey goals. Section 5 describes the plan and outlook for Y4 and Y5.

\section{THE DES Y1 TO Y3 SURVEY PROCEDURES}

\section{Observation Schedule \& Staffing}

The DES observations are staffed at the telescope by collaboration members. The Operations Scientist schedules the observing team from among the volunteers. There are three observing roles during full nights. "Observer 1" controls the camera through the data-acquisition interfaces [3] and executes the nightly program by following standard DES observing procedures [16]. This observer ensures that images are being recorded, pays attention to the alarms and warnings, and solves routine problems where procedures have been established. "Observer 2" performs quality control procedures, checks the exposures for problems and ensures that the image quality is as expected given the current conditions. The "Run Manager" is the lead observer and is responsible for ensuring that the two other observers understand how to perform their roles. Indeed, though observers are asked to read online procedures in advance of their observing trips, training is performed on site by the Run Manager. The Run Manager also has some daytime responsibilities described below, so they are not expected to stay up for the full night. During halfnight observations DES usually has only two observers with the Run Manager taking on one of the roles. Having a well-staffed and well-rested observation team reduces the chance for mistakes and misunderstandings that cost observing efficiency.

Generally, the "Observer 1" role can be performed by an inexperienced but attentive observer. We often fill that position with students and post-docs who have no previous observing experience. We prefer to have more- 
experienced observers as "Observer 2" for their expertise in image quality assessment. Run Managers are required to have performed both observing roles effectively because of their responsibility.

Support is available to the observers through the CTIO Telescope Operator (on hand), the CTIO Observer Support Specialist (on-site), the CTIO Instrument Scientist (typically by phone), and the DES Operations Scientist \& Support Team (by internet connection).

\section{Daily Operations Cycle}

The typical "Daily Cycle" starts with the Run Manager's Meeting held daily at 16:00 CTIO time. The Run Manager meets by phone with the Operations Scientist \& Support and Data Management (DESDM) Teams. We discuss any technical or procedural problems that occurred during the previous night, provide additional information to DESDM about individual images that might be problematic (for example, if the telescope slewed during the image), receive the DESDM-calculated data quality from previous night's imaging, and discuss what to expect from the "Observing Tactician" (OBSTAC) (see next subsection) based on the expected weather conditions. We discuss any unusual procedures that the observers might need to execute, such as Target-of-Opportunity (TOO) observations, or (somewhat less frequently) reactions to current camera or telescope hardware conditions. After this meeting the Run Manager implements the data quality, updating the Exposure Table so that OBSTAC has up-to-date information on which images need to be redone.

The period before twilight is used for calibrations and to establish the basic functionality of the instrument. An LED system [17] illuminates a flat-field screen attached to the inside of the dome. We take a set of biases and flats in each of the filters. These images are used in the daily calibration. An hour before sunset the telescope operator will open the dome. At minus 10 degree twilight (roughly 40 minutes after sunset) the observers execute three standard star field [18] exposure scripts, one at high airmass $(X=1.65$ to 2.1), one at medium airmass $(X=1.25-1.65)$, and one at low airmass $(\mathrm{X}<1.25)$. The standard star fields are fields of stars with previously calibrated brightnesses in each of the DES filter bands. They are used for characterizing that night's instrumental and atmospheric response (by fitting the observations to a set of "photometric equations") and are an integral part of the photometric calibration for DES. At minus 12 degree twilight (roughly 48 minutes after sunset) the observers begin OBSTAC observations. These continue during the night until minus 10 degree morning twilight, for standard star observations, and finally dome. Observer 1 controls SISPI and makes sure exposures are being recorded as expected. Observer 2 maintains a watch on the data quality using streamlined image analysis tools such as "Quick Reduce" $[3,16]$ developed by DES-Brazil and KENTools [16].

The observers maintain commentary and notes in an electronic logbook. At the end of each night the observers create two night summaries. The "CTIO Night Report" lists weather conditions, problems encountered, and the fraction of time lost to each. The "DES Night Summary" provides the narrative of the shift including the expected plan with ephemeris, the conditions, accomplishments, problems encountered, and notes for the DESDM team. A series of automatically generated plots and statistics follows, including transparency, PSF, and ellipticity of stars for each image, a note of any gaps between exposures of greater than 60 seconds, the progress on wide-field and SN surveys, and a list of exposures. This summary provides a concise history of what happened during that particular night.

The images are transferred by the NOAO Data Transport System [19] (DTS) to NCSA/UIUC in UrbanaChampaign, Illinois, usually within 5 minutes after the moment that the shutter closed. Copies of the data are stored in La Serena and at the NOAO Science Archive in Tucson [20]. To conserve both disk space and network bandwidth the DECam data are losslessly FITS tile-compressed [21] using FPACK. Note that both the DESDM pipeline, described below, and the Community Pipeline [22] use a lossy FPACK compression [23].

The camera and telescope are returned to a safe condition after observations are finished.

\section{OBSERVING TACTICIAN (OBSTAC)}

During the course of the season a variety of weather, seeing, and sky-brightness (Moon) conditions are expected to occur. A computer application, the "Observing Tactician" (OBSTAC) [24] uses this information to select the highest 
priority fields to observe during the next short (5-15 minute) interval throughout the night. A simplified version of the OBSTAC decision tree is shown in Figure 3. If any of the time-domain fields have not been observed in the past 6 nights, then OBSTAC selects the field with the longest gap to minimize deviation from the desired time-domain cadence. If there are time-domain fields that have not been observed in the past 3 nights, and the seeing is too poor to take exposures useful for weak-lensing ellipticity measurements but sufficient for SN photometry, then these are observed, resetting the cadence at minimal cost to wide-survey progress. OBSTAC will then select among not yet completed wide-survey exposures (tracked by an exposure table in the SISPI database) that are likely to be of acceptable quality (based on predicted airmass, seeing, and sky brightness).

To ensure that exposures on the western side of the WF footprint are completed in the beginning of the DES season (the only time they are observable at acceptable airmass) and that as few exposures in the eastern side of the footprint are completed before the end of the DES season (when no other exposures are possible), OBSTAC generally prefers exposures in fields that set earlier in the night. In DES Y1 and Y2, observations in the WF footprint were prioritized according to sidereal time at which the "observable field" set with an airmass limit of 1.4. In Y3 this was modified as describe below (Section 4). Among exposures with the same priority, OBSTAC selects exposures that minimize slew time.

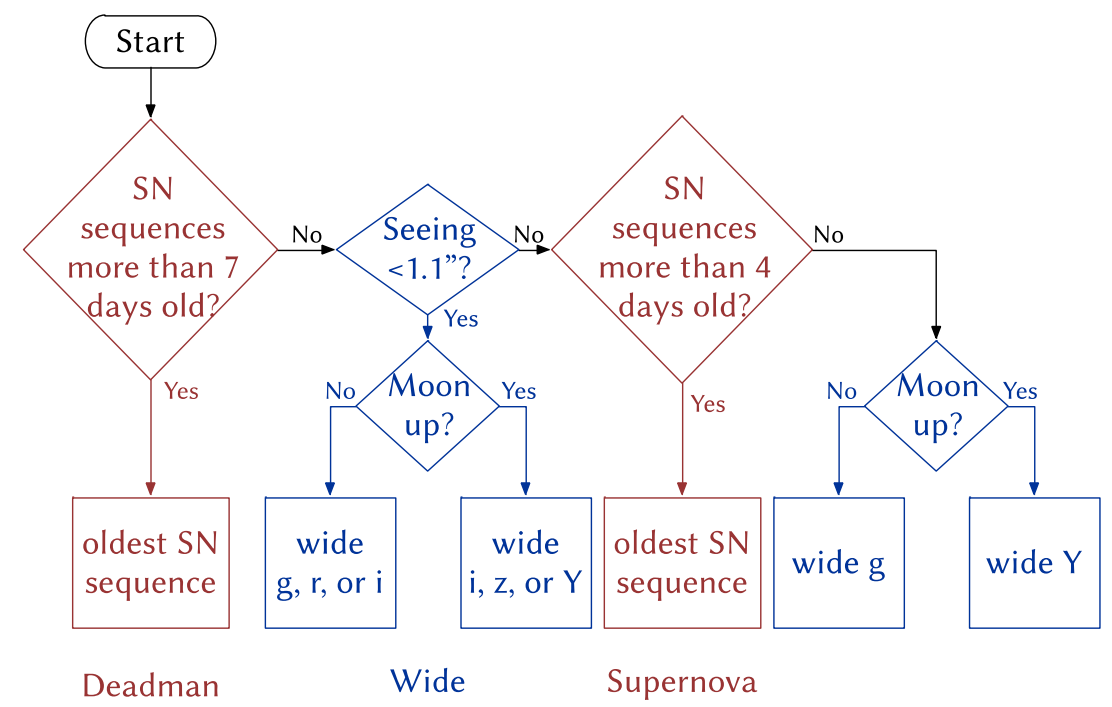

Figure 3 A slightly simplified version of the scheduling algorithm implemented by the Observing Tactician algorithm "OBSTAC". The "seeing" is that expected based on current conditions when projected to an equivalent i-band observation taken at zenith. "wide" is short for "wide-field" observations. Priorities can be adjusted for tiling \#, RA \& DEC, etc ... in the wide field survey. From left, the horizontal choices are loosely labeled "condition 1" for "deadman SN", "condition 2" for good PSF WF, "condition 3" for poor PSF SN observations should any sequence not have been observed in the previous 4 nights, and "condition 4" if the PSF is poor and the SN are all already up-to-date.

\section{Data Processing \& Data Quality}

The DESDM pipeline [25] performs image detrending and calibration that we referred to as "First Cut" in order to assess the quality of each exposure with respect to the minimum requirements necessary for DES to obtain its scientific objectives. The overscan and bias is subtracted and the image is divided by the mean dome flat. The CCD crosstalk is removed using a premeasured matrix, a linearity correction applied, fringe and pupil ghost corrections are combined, and a star flat is applied to subsections of each CCD. An astrometric solution for each image is found by comparing to known stellar positions in the $4^{\text {th }}$ USNO Astrograph Catalog (UCAC-4) [26]. Finally, the point spread function (PSF) is determined by examining the shapes of stellar images and then the position, brightness, and rudimentary shape of objects detected in each image are cataloged. 
We determine if the image is adequate for the wide-field survey by requiring that the "Effective Exposure Time" [27], $\quad t_{\text {effective }}=(0.9 \mathrm{k} / \mathrm{FWHM})^{2}\left(\mathrm{Bkgd}_{\text {dark }} / \mathrm{Bkdg}\right)\left(10^{-2 \mathrm{C} / 2.5}\right)$ exceeds a minimum. Here $\mathrm{k}$ is a filter-dependent "Kolmogorov Factor" scaled relative to i-band that takes into account the natural seeing dependence on wavelength, FWHM is the delivered point-spread-function for stars, Bkgd and Bkgd dark $_{\text {are }}$ the measured sky background and dark sky condition, and $\mathrm{C}$ is the atmospheric extinction offset calculated from a comparison of the brightness of stars within the image to those in the APASS DR7 and/or NOMAD public catalogs [28-29]. $t_{\text {effective }}=1$ corresponds to an exposure taken at zenith with no moon, clear skies, and nominal seeing, and sky brightness, and atmospheric extinction. We require that $t_{\text {effective }}>0.2$ for $\mathrm{g}$, and Y-bands and $>0.3$ for $\mathrm{r}, \mathrm{i}$, and z-bands. Each image is then checked for artifacts, such as satellites and airplane trails, and these images are flagged.

Supernovae are discovered by searching for temporal variations in brightness between SN template images and SN exposures. These SN search images are first processed through a detrending pipeline similar to First Cut, and then through a difference imaging pipeline [15]. The SN difference imaging pipeline aligns the template and search images, adjusts the template image to match the seeing conditions of the search image, then subtracts the two images to produce a "differenced" image. Object detection software runs over the differenced image to identify transient objects, these transient objects are then passed through machine learning algorithms [30] to identify transients which are supernova candidates. The data quality and the efficiency of this pipeline is monitored by inserting fake supernovae into the search images and monitoring how well these fake events are recovered by the pipeline. In particular, four fake supernovae of fixed magnitude (magnitude 20) are inserted into each CCD in each search image. The search images are considered to be of acceptable quality if $>90 \%$ of the fixed magnitude fakes is recovered and the $\mathrm{S} / \mathrm{N}$ ratio of the magnitude 20 fakes is $>20$ for shallow fields and $>80$ for deep fields. There is an additional requirement that seeing is $<2.0$ arc seconds if projected to $i$-band at zenith instead of the filter/airmass combination of the exposure.

The First Cut processing and data quality evaluation and the SN image pipeline are typically turned around in less than 24 hours. The results are applied to the Exposure Table by the Run Manager as described previously.

\section{DECAM MAINTENANCE AND UPGRADES Y1 TO Y3}

Generally the camera and telescope performance has been very good since SV. Nonetheless, there has been a continuous effort [31] to improve it. This work is carried-out by technical staff from both Fermilab and CTIO.

Prior to and during Y1, "hardening" of camera infrastructure systems against unexpected failures was done. This work has been discussed extensively in the "DECam Paper" [3] and elsewhere [7]. Repair of the dome floor and primary mirror cooling system temperature occurred in Nov. 2013, partway through Y1. Photodiodes were mounted on the top end of the cage to protect the CCDs from being exposed during very bright conditions by providing an external interlock to the shutter. Studies of stray light during commissioning led to retrofits to the shutter and filter changer assemblies [3] completed in March 2014, after Y1. DES arranged the regular servicing of the filter changer mechanism and the shutter at that time. A community use VR-band filter [3] was added to the camera at the same time.

The changes during Y2 were directed towards improving the DECam image quality. By Sept. 1, 2014 the primary mirror air pad controls were upgraded to more precise ones. There were a couple of changes to the active optics system (AOS) controls [3]. It is necessary to describe how the AOS works [32-33] in order to describe the changes. The hexapod, which mechanically couples DECam to the Prime Focus Cage, provides position adjustment for DECam with 5 degrees of freedom with respect to the primary mirror: translation, piston, tip \& tilt. A look-up-table (LUT) provides the nominal hexapod position as a function of temperature, Hour Angle, and Declination. The AOS then uses out-of-focus stars on CCDs located above and below the nominal focal plane to supply a position correction to the hexapod, based on analysis of the just-readout exposure, in time for the next one. Analysis of all DECam exposures led to a more accurate default hexapod position that was introduced Oct. 21, 2014. An improved LUT was installed on Jan. 4, 2015, towards the end of Y2. These changes led to an improvement of about 0.25 " to the PSF, to be taken in quadrature with all the other contributions [3].

Prior to Y3 (July 2015) there was continued work on the $\mathrm{LN}_{2}$ system that cools the camera including the replacement, for the $5^{\text {th }}$ time, of the pump that provides $\mathrm{LN}_{2}$ to cool the CCDs. We continued to rotate between two 
pumps, removing one and installing another. The one that is removed is refurbished at Fermilab, where we replace the bearings on the rotor shaft and rebalance the rotor. We are studying the pump to see if we can improve the lifetime to more than 12 months. A change in the material for the rotor beating from Teflon plus $\mathrm{MoS}_{2}$ to a more durable polyimide-based plastic might do the trick. That is currently under test at Fermilab. Two of the vacuumjacketed $\mathrm{LN}_{2}$ transport pipe segments were replaced with new ones that were more robust and also easier to install/remove. Prior to and after these changes the $\mathrm{LN}_{2}$ system, which is designed [3] to be closed-loop with more than $100 \mathrm{~W}$ of cooling headroom, the system was still venting $\mathrm{N}_{2}$. Engineering analysis indicated that the likely causes were: a heat leak due to a poor vacuum in one of the new vacuum-jacketed lines (1r), less than ideal operation (water-glycol coolant too cold) of the two He cryo-coolers, and wear of the two He cryo-coolers, which had been in nearly continuous operation for about $3 \frac{1}{2}$ years. When these were mitigated during the $\left(6^{\text {th }}\right)$ pump change in Feb. 2016, after Y3, the $\mathrm{LN}_{2}$ system operated in closed-loop mode with $140 \mathrm{~W}$ of cooling headroom. The vacuum in the 1r line has degraded over the past 4 months from a few mTorr to $\sim 100$ mTorr. The cooling headroom is now down to about $100 \mathrm{~W}$. At least the CTIO staff doesn't have to fill it.

Partway through Y3 mountaintop dust was discovered on the inner and outer surfaces of $\mathrm{C} 1$, the lens that is closest to the primary mirror. This summarizes the report from CTIO. The dust was on approximately one third of the outside surface in the direction towards the SE in primary mirror coordinates. It is also on the complete interior surface. Bowl-shaped, that surface is downwards when the camera is oriented to zenith. It has been accumulating since installation, almost surely anytime a strong wind blows directly onto $\mathrm{C} 1$. In very windy conditions we have seen the wind overcome the dry-gas purge as that vents from $\mathrm{C} 5$ to $\mathrm{C} 1$, escaping out of narrow ports between the lens cells and the lens. There was no evidence for dust on any other lens surfaces. Figure 4 shows some photographs of $\mathrm{C} 1$ that highlight the dust. The dust was removed during the Feb. 2016 shutdown, after Y3. There followed a 4\% gain in throughput.
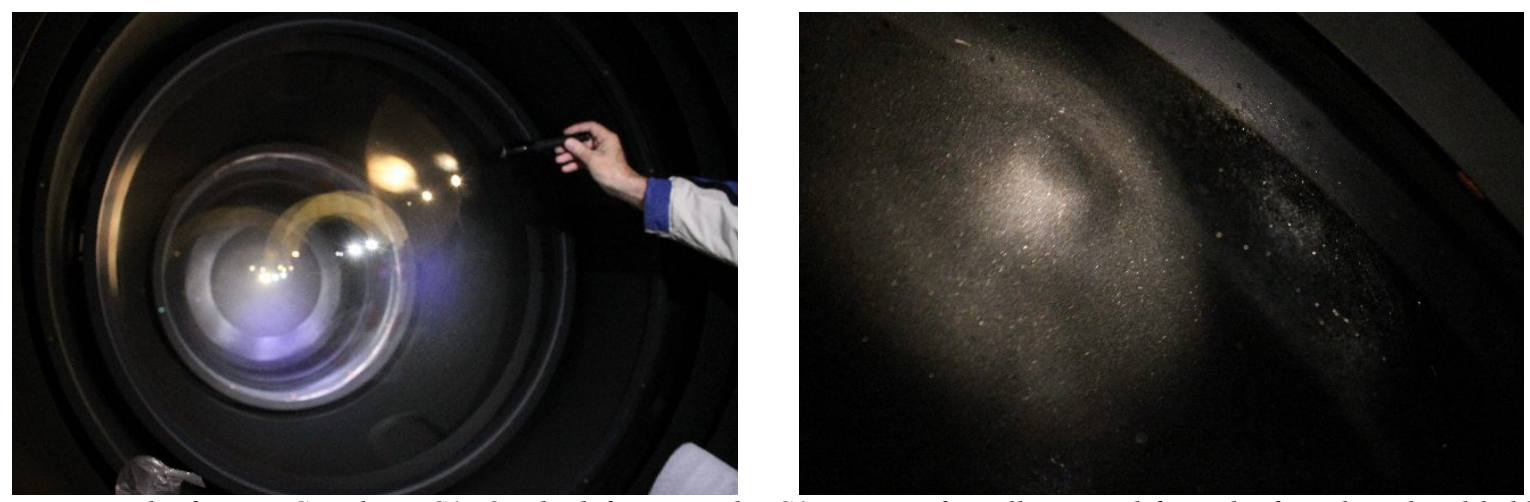

Figure 4 The first DECam lens, C1. On the left we see the C1 outer surface illuminated from the front by a hand-held flashlight. We can see through C1-C3, up to the block filter. On the right we see the dust, highlighted by changing the angle of the light. This dust was removed after Y3 (during Feb. 19 to 24, 2016). Photos from CTIO staff.

\section{THE DES Y1 TO Y3 NARRATIVE, EFFICIENCY, LOSSES, AND PROGRESS}

\subsection{Y1 WF Survey}

For Y1 we chose to observe the SN fields plus a 2000 square-degree subset of the entire DES WF footprint consisting of the first four tilings in each of the five filters, g, r, i, z, and Y-bands. This choice for the WF survey provided deeper and more uniform observations over that subset of the DES field than we would achieved had we tried to cover the full $5000 \mathrm{WF}$. That choice of depth over total area enabled and benefited science analyses and publications, allowing them to be performed earlier, albeit on a subset of the WF area.

The first Y1 night of DES observing started on the night of August 31, 2013. There were 91 full nights through January 4, 2014 and 28 first-half nights after that, for a total of 105 nights. Y1 concluded during the night that started on February 9, 2014. See Table 2. The western part of the part of the survey field was no longer observable by the end of the run and the eastern part of the survey field was setting in the first half of the night by the end of January. Other nights during Y1 were assigned to "Engineering", around periods of the Full Moon, and to "Community Users" for the remaining 24 full nights and 28 second-half nights during Y2. 
We track efficiency using a combination of information including the ELog, the DES and CTIO Night Reports, and tools. The DES and CTIO Night Reports were collated to produce the estimate of how we spent our time. See Table 3. A sophisticated query of the exposure database makes separate summations of the exposure lengths and gaps between exposures to provide a "shutter-open efficiency". That calculation indicated we performed OBSTAC observing with the shutter open for $63 \%$ time. The remainder of time is spent with the shutter closed and either reading out the CCDs or moving the telescope and/or dome. The time between shutter-closed and shutter-open was $26 \mathrm{~s}$ to $30 \mathrm{~s}$ when there was no telescope slew. If there was a slew to a new position, the slewing and settling-time of the telescope, rather than the readout of the CCDs, determined when we could next open the shutter.

During Y1, the time lost due to the camera or telescope failures was dominated by two incidents. A night was lost during October 2013 due to a software error in the Telescope Control System. A night was lost during November 2013 when it was realized that a CCD on the focal plane was not responding correctly to clock signals. Subsequent investigation showed that it had failed. These and other, minor, problems are documented elsewhere $[3,7]$. The largest source of lost observing time was the weather. Table 3 indicates 90 1/4 hours $(10.2 \%)$ were lost during Y1 because the weather was too poor to open the dome.

Table 2 Scheduled start and end dates for DES observing, and the number of half-nights and full nights for Y1 to Y3.

\begin{tabular}{|c|c|c|c|c|c|}
\hline Season & Start Date & End Date & $2^{\text {nd }}$ Half Nights & Full nights & $1^{\text {st }}$ Half Nights \\
\hline "Y1" & Aug. 31, 2013 & Feb. 09, 2014 & 0 & 91 & 28 \\
\hline "Y2" & Aug. 15, 2014 & Feb. 15, 2015 & 10 & 80 & 41 \\
\hline "Y3" & Aug. 04, 2015 & Feb. 12, 2016 & 32 & 73 & 39 \\
\hline
\end{tabular}

Table 3 DES Operational efficiency sums accumulated through Y3. These are based on the observer's reports in the CTIO Night Summaries. "Time Available" is the time we should spend observing." Observing Time" is the number of hours the observers were actually engaged in observing. "Engineering Observations" are those in service to the understanding of camera or telescope systematics. Next is indicated the number of hours lost to bad weather that results in closing the dome instead of observing, to a failure of the telescope, dome, or mountaintop infrastructure, to the camera, and to obvious observer error ("Other"). In the case of the lattermost, increased training and/or a clarification to the procedures generally prevents any reoccurrence.

\begin{tabular}{|c|c|c|c|}
\hline Operations & $\begin{array}{c}\text { DES Yr. } 1 \\
\text { Accumulated } \\
\text { Hrs. (\%) }\end{array}$ & $\begin{array}{c}\text { DES Yr. } 2 \\
\text { Accumulated } \\
\text { Hrs. }(\%)\end{array}$ & $\begin{array}{c}\text { DES Yr. } 3 \\
\text { Accumulated } \\
\text { Hrs. }(\%)\end{array}$ \\
\hline Observing Time Available & $888 \frac{1}{4}(100 \%)$ & $9283 / 4(100 \%)$ & $9693 / 4(100 \%)$ \\
\hline Observing Time & $7511 / 2(84.6)$ & $7821 / 2(84.2)$ & $6361 / 2(65.6)$ \\
\hline Engineering Observations & $0(0)$ & $0(0)$ & $13 / 4(0.1)$ \\
\hline Bad Weather & $901 / 4(10.2)$ & $140(15.1)$ & $2933 / 4(30.3)$ \\
\hline $\begin{array}{l}\text { Telescope or Infrastructure } \\
\text { Failure }\end{array}$ & $18(2.0)$ & $27 / 8(0.3)$ & $28(2.9)$ \\
\hline Camera Systems Failure & $253 / 4(2.9)$ & $31 / 8(0.3)$ & $93 / 4(1.0)$ \\
\hline Other & $23 / 4(0.3)$ & $1 / 4(0)$ & $0(0)$ \\
\hline
\end{tabular}

During Y1 we recorded $17671 \mathrm{WF}$ images. Of those, $82 \%$ (14444) passed the $t_{\text {effective }}$ data quality measure. Of the $18 \%$ that were declared "unusable", the bulk were generally g- and Y-band exposures directed by OBSTAC during poor achieved seeing that were recorded before the changes to the dome floor and primary mirror cooling mentioned above. As a result of OBSTAC's scheduling g- and Y-band WF exposures when the seeing is poor, the achieved 
PSF in the good (passed the teffective DQ criterion) g- and Y-band exposures had a median FWHM of 1.17". The good r-, i-, and z-band exposures (RHS) had a median seeing of 0.94". The survey goal in this latter set of filters is a median seeing of 0.90 " or better. Figure 5 shows a map of the number of tilings with good exposures in each of the 5 filters. It shows that we successfully covered the eastern part of the Y1 field with four tilings in all five filters. It also shows that there are survey areas in the western part of the Y1 field where we did not achieve four tilings in each filter (because of the early seeing struggles), that there are areas in Stripe 82 where we did not achieve any tilings in dark-time filters (due to the proximity of the Moon), and that there is a considerable part of the eastern Y2 area that was finished in $\mathrm{Y} 1$, especially in $\mathrm{z}$ - and $\mathrm{Y}$-band (because we ran out of accessible $\mathrm{Y} 1$ targets in the latter weeks of the Y1 observing season).

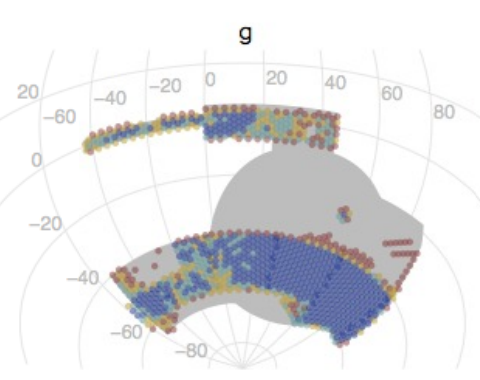

$\mathbf{z}$

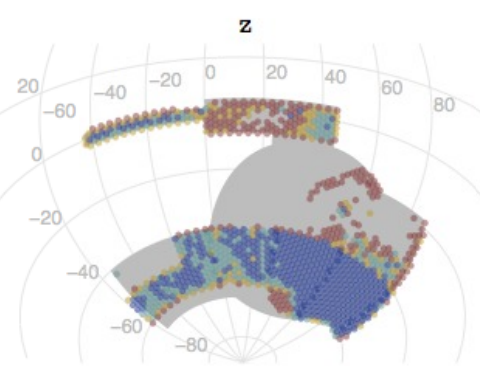

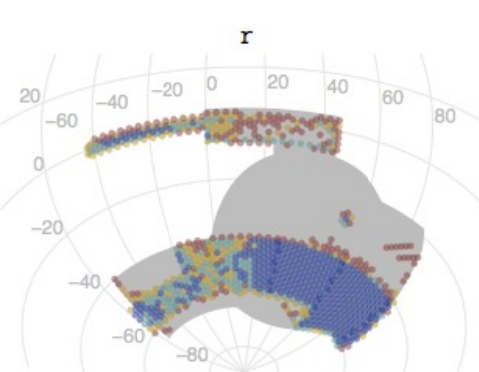

Y

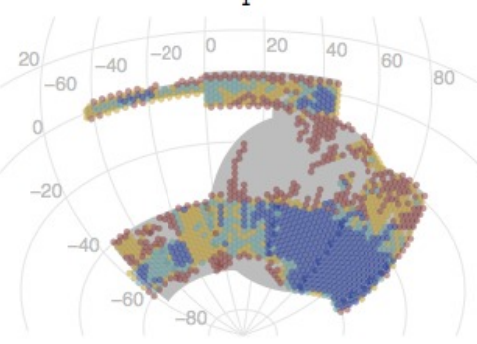

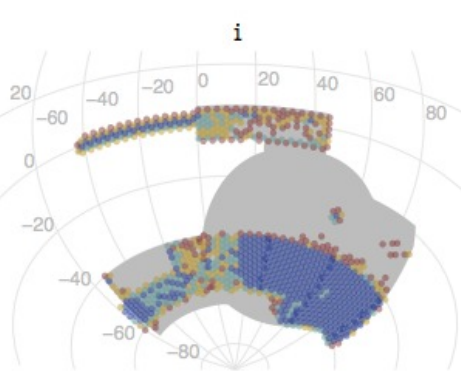

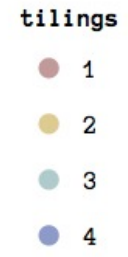

Figure 5 The Y1 survey fields include Stripe 82 (at the Equator), and the boxed-in area at roughly $-40<R A<-60$ degrees. We scheduled four tilings in each filter. The colored dots represent the number of "good" exposures (as defined by the $t_{\text {effective }}$ data quality criterion described above) achieved in each filter. Grey-colored fields are unobserved.

\subsection{Y2 WF Survey}

For Y2 we chose to observe the SN fields plus the remainder of the DES WF footprint up through the first four tilings/five filters that was not observed in Y1. That is, we planned to finish the unobserved parts of the Y1 field and observe the complement of the field outlined in Fig. 2 and filled in light blue color. Detailed simulations performed to develop the optimum schedule for the Y2 observing schedule took into account variants in the weather based on historical data, expected data quality, the distribution of bright and dark time, and included constraints on the total number of nights and balance of bright/dark time. The metric used for evaluation was the total number of completed survey quality (good) exposures. The resulting schedule from CTIO was reasonably close to our request. We started in mid-August. In late-August we transitioned to full nights. In late-December we transitioned to $1^{\text {st }}$-half nights and continued until mid-February 2015. The schedule differed from Y1 in that we started a little earlier had significantly more half nights, an improvement because $1^{\text {st }}$ half-nights early in the season and $2^{\text {nd }}$ half-nights late in the season were less useful due to the location of the survey footprint. Again, see Table 2 for details.

Y2 went very smoothly. The camera and telescope performed very well and very reliably. The only difficulty was the weather, for dome-closed downtime was 50\% worse than during Y1. See Table 3. During Y2 we recorded $17788 \mathrm{WF}$ images. Of those, $81 \%$ (14483) passed the $t_{\text {effective }}$ data quality measure. The good exposures were more evenly divided among the 5 filters than occurred in Y1 simply because OBSTAC didn't go off into the poor PSF WF condition as often. Because OBSTAC didn't often force g-band or Y-band observing because of poor achieved 
seeing (unlike Y1), the achieved PSF in the good g- and Y-band exposures had a median FWHM of 1.07" and 0.90", much better than Y1. The good r-, i-, and z-band exposures had median seeings of 0.98", 0.92", 0.90". Figure 6 shows the WF survey progress as of the end of Y2. It shows that we finished the Y1 field and except for a hole at RA 30 deg, DEC -30 degrees, l finished the Y2 field through 3 tilings. It also shows that in the eastern side of the field we got a bit ahead of $\mathrm{Y} 2$ in the Y-band.

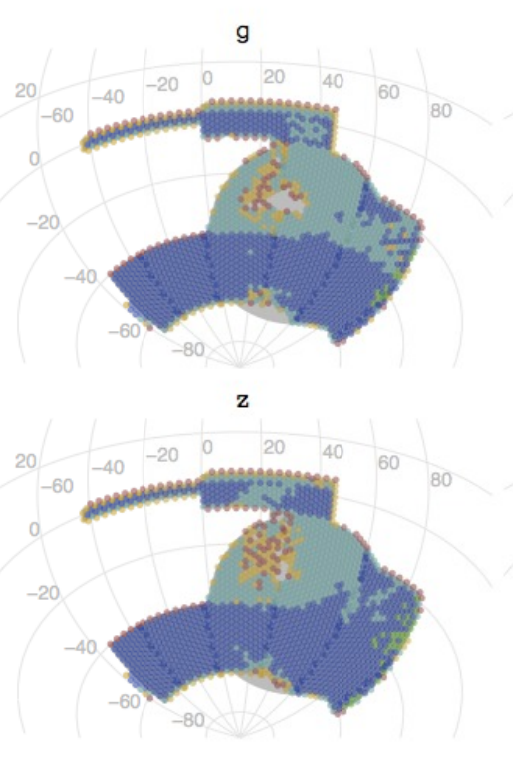

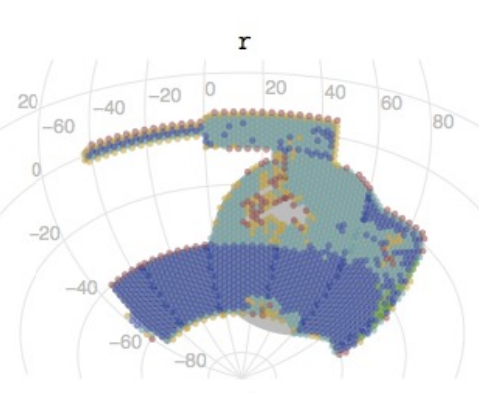

Y

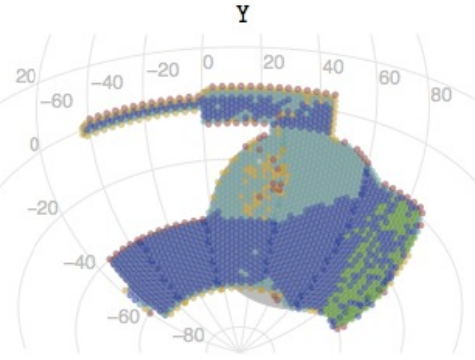

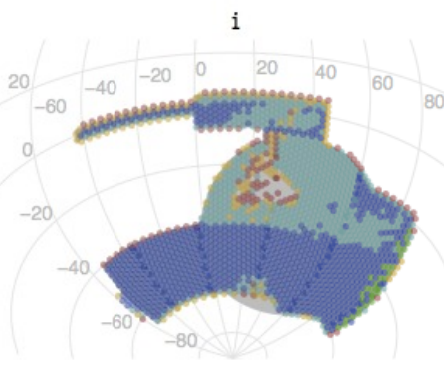

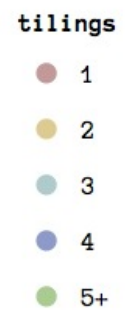

Figure 6 The Y1-Y2 WF survey observations included the fields that were not observed in Y1 with the goal of observing 4 tilings in each filter. The colored dots represent the number of "good" exposures (as defined by the teffective data quality criterion described above) achieved in each filter. Note that by the end of Y2 we have a region where we haven't taken any exposures (the center of the fat part of the field), and some areas where we have 5 or more good exposures (particularly in the Y-band, east side).

\subsection{Y3 WF Survey}

For Y3 we chose to observe the SN fields plus the entire DES WF footprint up through the first six tilings/five filters that we not previously observed. We assigned higher priority to tilings 1-4 than to tilings $5 \& 6$. We planned to start two weeks earlier in the season than during Y2, because it was to be Moonless in early August, and with even more emphasis on half nights than Y2 because the simulation (described above) advised us that was optimal. See Table 2.

During Y3, the OBSTAC strategy was slightly modified to adapt to our having so many half-nights, which relieved the some of the pressure to observe to the west, and to fill the unobserved hole at about $(30,-30)$ in Figure 6 , which would have otherwise fallen further behind because we would have been observing west when this central area was transiting. During Y3, therefore, OBSTAC preferred areas of the footprint near transit when the sidereal time was earlier than 2 hours (30 degrees). After this time, OBSTAC prioritized exposures west of R.A. $=30$ degrees by closeness to transit, and east of 30 degrees by setting time. We eventually also prioritized tilings $1-5$ over tiling 6 so that Y1 and Y2 tilings would likely be completed.

We also had an interesting Target of Opportunity (TOO) arrangement with another project [34-35] whereby 3 nights were added to our 105 nights. The TOO was expected to use those 3 nights at times of their choosing from among the DES allocation, coordinated in the daily "4 o'clock Meeting" and subject to the restriction that the TOO observations would have to work around any DES SNae observations that were in the OBSTAC "deadman" status indicated in Figure 3. The arrangement provided that the 3 nights could be selected piecemeal, with cadences as short or long as needed for their program.

Y3 had a dismal start. The first nine nights were simply clouded out, culminated by a blizzard that dumped 2 feet of snow on the mountain and knocked out electrical power. We got out first on-sky observations on August 13th. 
Going forward, the weather never was rarely close to the seasonal average and by now you have seen Table 3, which shows we lost $\sim 30.3 \%$ of our possible observing time with the dome closed. The weather pattern was diagnosed as a strong El Niño Southern Oscillation [36]. The telescope and camera performed reasonably reliably (note that the knocking out of electrical power for a few days during the August blizzard was the biggest contributor to infrastructure failure). The time 9.5 hours lost due to camera problems was primarily in two incidents. On one night we lost about 3.5 hours when the readout crate cooling tripped off, the crates overheated and shut themselves off as a protective measure, and then complications ensued trying to restart. On another occasion the beginning of the evening start of SISPI didn't go smoothly until we attained expert help, costing about 3 hours. During Y3 we recorded $13433 \mathrm{WF}$ images. Of those, only $71 \%$ (9565) passed the $t_{\text {effective }}$ data quality measure. The reason that a higher fraction of images were declared bad was extinction due to cloudy weather. Y3 ended the night of February 12, 2016. The good r-, i-, and z-band exposures (RHS) had a median seeing of 1.02", 0.95 ", and 0.92", a little worse than either Y1 or Y2. Figure 7 shows he survey progress map as of the end of Y3. That figure shows that we successfully covered the eastern part of the Y1 field with four tilings in all five filters. It also shows the most of the survey area is covered to 4 or 5 good tilings. An exception is Y-band, where the western side is completed through 6 tilings. The "Y2 hole" is filled in. However, falling behind $1 / 10^{\text {th }}$ of a season in $\mathrm{Y} 1$ and $\mathrm{Y} 2$, and $40 \%$ of a season in Y3, we have completed about $80 \%$ of that originally planned for the end of Y3 given average conditions. The principal reasons are broken dome and primary mirror cooling increased the sensitivity to weather-related temperature swings (identified and solved during Y1), bad weather (Y2), and extremely bad weather (Y3). Figure 8 indicates that the $\mathrm{Y} 3$ weather was the worst recorded at CTIO and that is was unpredictably poor.

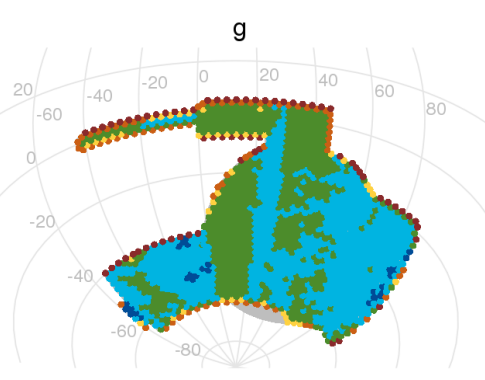

Z

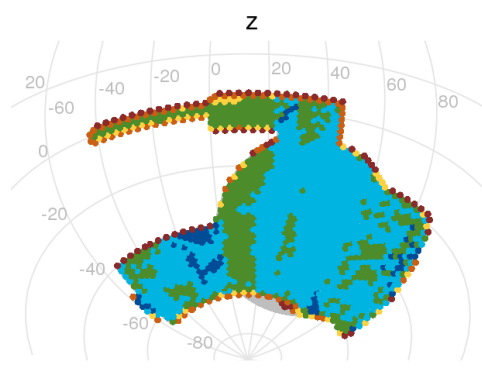

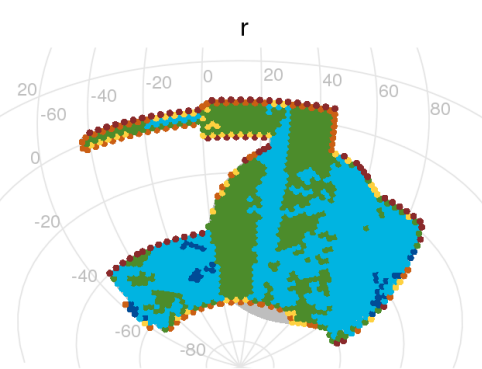

Y

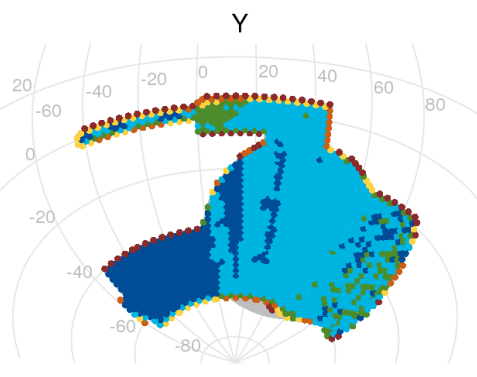

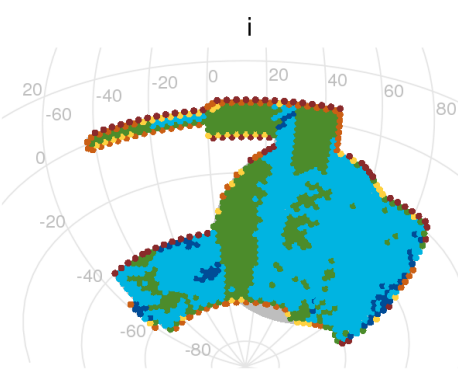

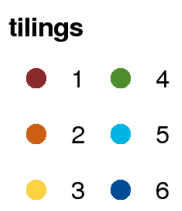

Figure 7 The completed Y1-Y3 survey fields in each filter. The colored dots represent the number of "good" exposures (as defined by the $t_{\text {effective }}$ data quality criterion described above). Note that the "Y2 hole" is filled in. The odd striping in RA is due to the change in OBSTAC priority for Y3 described above. 


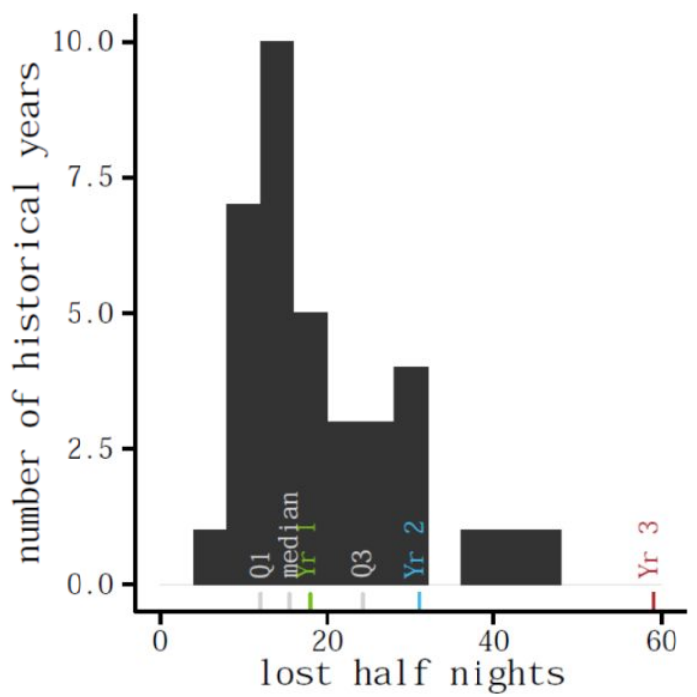

Figure 8 Illustrates the distribution of lost half nights during simulated DES observing seasons using the historical weather data from CTIO during the relevant periods. The data from Y1, Y2, and Y3 is marked on the chart as well. This indicates Y1 was close to the median. Y2 was worse than $\sim 80 \%$ of simulated seasons. There was no precedent for weather as poor as that which we got in $Y 3$ in the 31 years of historical data.

\subsection{Y1 to Y3 SN Surveys}

The Y1 through Y3 DES Supernovae Surveys were interleaved with and were subject, of course, to the same conditions as the corresponding WF survey. SN observations were taken according to the prescriptions of the OBSTAC algorithm shown in Figure 3. Generally that results in a SN observations being taken under the six night gap "deadman" condition 1 (highest priority), or under the poor PSF condition 3 if some SN field has a 4 night gap and the projected PSF at zenith in r-band would be $>1.1$ " (for WF observations would not pass the WF survey DQ test anyway, so we might as well take SN observations). We successfully observed each SN field 20 to 28 times in a DES season.

\section{Y1 SN}

We recorded 2699 SN-sequence exposures during Y1. Of these 95\% passed the SN data quality threshold described in Section 2. Figure 9 shows the nights on which we observed each SN sequence for the 10 SN fields. These gaps are summarized in Figure 10, which shows the distribution of nights between successful sequences for the shallow and deep SN fields for Y1 to Y3. We note that when the weather is perfect these fields will be observed with typically six-night gaps. 30\% of the "shutter-open" time was for SN observations. We found roughly 1700 SN candidates of all types in Y1 [37].

\section{Y2 SN}

We recorded $2370 \mathrm{SN}$-sequence exposures during Y2. This is a bit less than Y1 because Y1 had so much condition 3 (poor PSF) SN observing. Of these 83\% passed the SN data quality threshold described previously. Figure 9 shows the nights on which we observed each SN sequence for the $10 \mathrm{SN}$ fields. These gaps are summarized in Figure 10, which shows the distribution of nights between successful sequences for the shallow and deep SN fields for Y1 to Y3. As during Y1, DES spent 30\% of “shutter open" time on SN observations during Y2.

\section{Y3 SN}

We recorded 2249 SN-sequence exposures during Y3. Of these 90\% passed the SN data quality threshold described previously. Figure 9 shows the nights on which we observed each SN sequence for the 10 SN fields. These gaps are summarized in Figure 10, which shows the distribution of nights between successful sequences for the shallow and deep SN fields for Y1 to Y3. Because of the poor Y3 weather made more expended periods of "bad" SN data, we spent $35 \%$ of our "shutter open" time on SN during Y3. Furthermore, because Y3 started early and spanned a time 4 weeks longer than Y1 and 2 weeks longer than Y2, the SN observations were to end early, so that we didn't expend 
a greater fraction of our overall observing time (105 nights) on SN data than in previous years. We ended the SN "deadman" OBSTAC condition 1 on January 21, 2016, just before a 15 night gap for engineering (around the Full Moon) and community observing. That would normally have meant the end of DES SN observations for the season. However, a few days after we restarted in February, the PSF conditions were poor enough that that the OBSTAC "SN in bad seeing" condition was triggered. Typical of Y3, the weather was so bad that we did SN observations even when we preferred not to have them anymore! Figure 9 and Figure 10 contain the plots for Y3, as well.
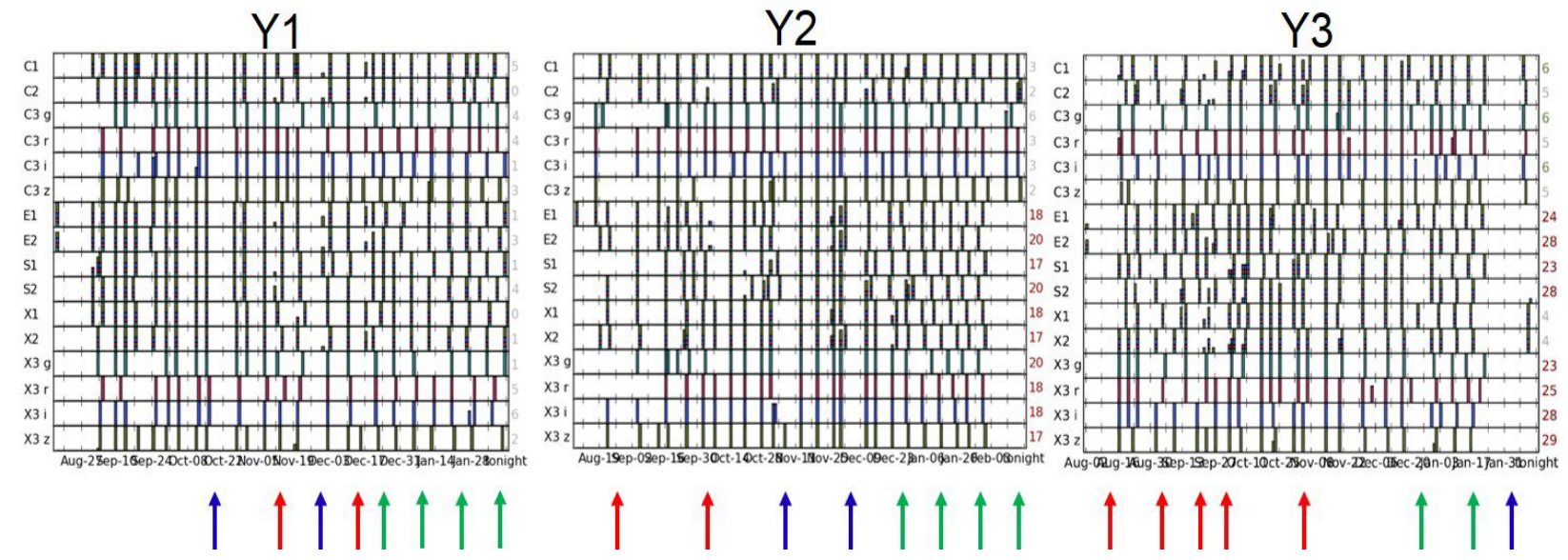

Figure 9 The DES Yearly SN observations, good data only, Y1 to Y3. The horizontal axis is the date from the beginning of the season to the end of the season. The vertical axis lists each of the 10 SN fields. If the SN field was observed there is a vertical bar with a separate color block for each filter. The two "deep fields" are C3 and X3. The arrows illustrate (red) periods with lots of bad SN data, (blue) long gaps as a result of a combination of schedule gaps and sometimes bad data, and (green) observations taken during long, steady periods of 6-night "deadman" OBSTAC conditions.

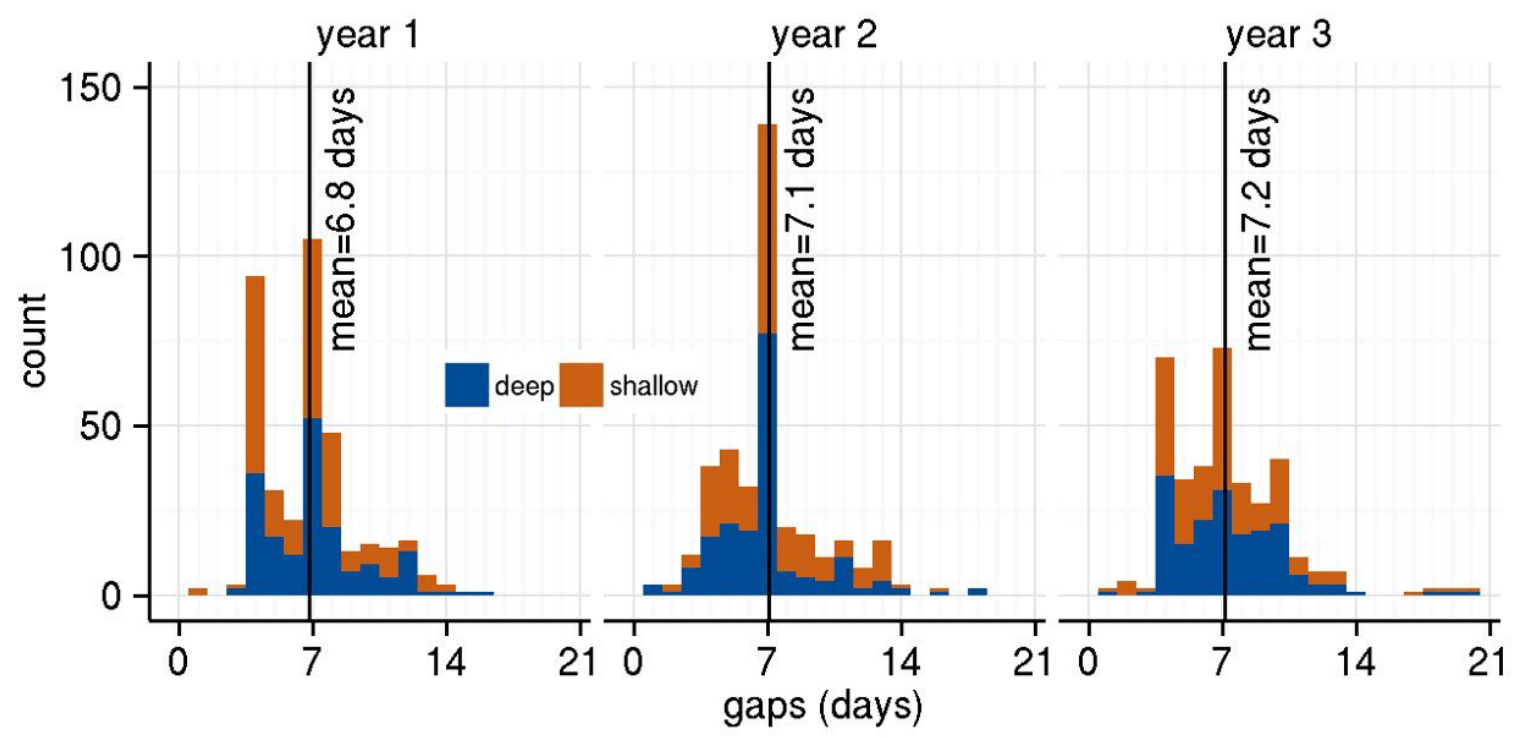

Figure 10 For Y1 to Y3, the number of nights between successful SN observations for the shallow and deep SN fields. For Y1, for instance, the peak at 4 night gaps is due to the frequent OBSTAC condition 3 of poor PSF, the peak at 6 night gaps is due to the OBSTAC "deadman" condition 1. During Y2 there was little of the $1^{\text {st }}$ peak. Ideally all of the observations are on the 6 night gap OBSTAC "deadman". Next to ideally there are no occurrences of more than 6 night gaps. The mean gaps for each season are indicated. 


\section{SURVEY YEARS FOUR \& FIVE AND OUTLOOK}

DES Y4 observing season is expected to start as usual, that is: sometime in August 2016 and finish in February 2017. Schedule details aren't available as of this time. The goal for WF observing will be to finish tilings 1-8 with priority given to those missed in previous seasons. We will make an adjustment to the WF survey strategy for the Y-band as follows. We will replace the last four Y-band tilings (7 to 10), which are 45 second exposures, with two tilings of 90 second duration exposures. In doing so we will use 3290 fewer exposures to gain approximately the equivalent Y-band depth, saving time for approximately 325 more 90 second exposures in either z-band or Y-band per remaining DES season. The $2^{\text {nd }}$ combined $(9+10)$ Y-band tiling will be given lower priority in Y4 so that we have sufficient Y-band tiling left to observe in Y5. We are hoping that Y4 is our first above-average weather season. It is predicted [36] that the surface temperature in the southern Pacific Ocean will drop, so we can hope for La Niña conditions by the time we start up again. We haven't made detailed plans for Y5 at this time as they, ultimately, depend on what happens during Y4.

For Y4 DES continues to look for ways to improve the image quality. We [38] used all of the images from the outof-focus CCDs (DONUT analysis) to improve the 5D active optics (hexapod) LUT and the primary mirror (4MAP) LUT. A new hexapod LUT, 4MAP LUT, and pointing LUT was produced and installed on May 25, 2016. The Blanco primary mirror support system (4MAP), as now implemented, presents forces to the primary depending on the gravity vector, plus a static astigmatism correction. This comes from a LUT within the telescope controls. Data taken in 2013 ascertained that it is possible [32] to improve the mirror figure as a function of sky position so as to better zero-out primary mirror aberrations (particularly astigmatism) through the analysis of the DONUT images and feedback of corrections between images though a PID loop to the 4MAP controls. Testing of this new algorithm, unprecedented for a wonderful old telescope, is scheduled for before the start of Y4. We expect an improvement to the best PSF exposures [39] of about 0.05 ".

Y5 is nominally the final season for DES observing. We've been thinking carefully about how well we can meet the science goals of the survey given that we are now $6 / 10^{\text {th }}$ of a DES season behind due to the unusually bad weather. Many analyses benefit from uniform homogeneous coverage of the WF survey area and the DES survey strategy has made that a priority from the start of Y1, adjusting OBSTAC along the way. If the weather during Y4 is a repeat of Y3, we will be roughly a full average DES season behind. It is not too early to think about whether or not we will need a Y 6 based on poor weather alone. After all, we didn't ask for a four year survey when we started the building the camera in 2008.

After DES, the DECam will continue to be available as a Community Instrument on the Blanco Telescope for a long time [40].

\section{SUMMARY}

The Dark Energy Survey Collaboration studies the accelerating expansion of the Universe through four complementary techniques. To produce the deep, 5000 square-degree survey and the 30 square-degree time-domain SN survey that are specified by the science goals, the collaboration designed and built the Dark Energy Camera, now operating on the Blanco Telescope at CTIO.

DES has completed the first three of five 105-night observing seasons. Operational procedures developed for Y1 subsequently tweaked and improved have achieved high survey efficiency. Though DES has successfully observed only $80 \%$ of an average first 3 seasons, primarily to bad weather, we do already have a reasonably uniformly covered WF survey with a least four good exposures in all five filters. In addition, the procedures and priorities dedicated to the 30 square-degree transient survey have provided the experiment with data containing light curves of $\sim 5000$ supernovae. DES has made good use of the initial data samples, with discoveries of new solar system objects [41], discoveries of dwarf galaxies and structure in the Milky Way [42-44], systematic studies of galaxy clusters [45-46], weak gravitational lensing [47-48], and our first cosmological results [49]. With more than 70 papers in some state of publication, there is every indication that DES will successfully complete its scientific agenda.

The start of Y4 is coming soon. Combined optimism about the weather with the planned improvements at the telescope leave us hopeful for our first above average DES observing season. 


\section{ACKNOWLEDGEMENTS}

We are grateful for the extraordinary contributions of our CTIO colleagues and the DES Camera and Operations Support teams in achieving the excellent instrument and telescope conditions that have made this work possible. We are grateful for the administrative and organizational assistance provided by Connie Lang (FNAL) and Ximena Herreros (CTIO).

Funding for the DES Projects has been provided by the U.S. Department of Energy, the U.S. National Science Foundation, the Ministry of Science and Education of Spain, the Science and Technology Facilities Council of the United Kingdom, the Higher Education Funding Council for England, the National Center for Supercomputing Applications at the University of Illinois at Urbana-Champaign, the Kavli Institute of Cosmological Physics at the University of Chicago, the Center for Cosmology and Astro-Particle Physics at the Ohio State University, the Mitchell Institute for Fundamental Physics and Astronomy at Texas A\&M University, Financiadora de Estudos e Projetos, Fundação Carlos Chagas Filho de Amparo à Pesquisa do Estado do Rio de Janeiro, Conselho Nacional de Desenvolvimento Científico e Tecnológico and the Ministério da Ciência, Tecnologia e Inovação, the Deutsche Forschungsgemeinschaft and the Collaborating Institutions in the Dark Energy Survey.

The Collaborating Institutions are Argonne National Laboratory, the University of California at Santa Cruz, the University of Cambridge, Centro de Investigaciones Energéticas, Medioambientales y Tecnológicas-Madrid, the University of Chicago, University College London, the DES-Brazil Consortium, the University of Edinburgh, the Eidgenössische Technische Hochschule (ETH) Zürich, Fermi National Accelerator Laboratory, the University of Illinois at Urbana Champaign, the Institut de Ciencies de l'Espai (IEEC/CSIC), the Institut de Física d'Altes Energies, Lawrence Berkeley National Laboratory, the Ludwig-Maximilians Universität München and the associated Excellence Cluster Universe, the University of Michigan, the National Optical Astronomy Observatory, the University of Nottingham, the Ohio State University, the University of Pennsylvania, the University of Portsmouth, SLAC National Accelerator Laboratory, Stanford University, the University of Sussex, Texas A\&M University, and the OzDES Membership Consortium.

The DES data management system is supported by the National Science Foundation under Grant Number AST1138766. The DES participants from Spanish institutions are partially supported by MINECO under grants AYA2012-39559, ESP2013-48274, FPA2013-47986, and Centro de Excelencia Severo Ochoa SEV-2012-0234.

Research leading to these results has received funding from the European Research Council under the European Union's Seventh Framework Programme (FP7/2007-2013) including ERC grant agreements 240672, 291329, and 306478 .

\section{REFERENCES}

[1] Flaugher, B., “The Dark Energy Survey”, Int. J. Modern. Phys. A20, 3121 (2005).

[2] Dark Energy Survey Collaboration, "The Dark Energy Survey: more than dark energy - an overview", accepted for publication in MNRAS, arXiv:1601.00329.

[3] Flaugher, B., Diehl, H. T., Honscheid, K. et al., "The Dark Energy Camera”, AJ 150, 150 (2015).

[4] Abbott, T. M. C., "Dark Energy Camera Installation at CTIO: Overview", Proc. SPIE 8444, 844445 (2012).

[5] Munoz, F, "Dark Energy Camera installation at CTIO - Technical challenges", Proc. SPIE 8444, 844446 (2012).

[6] Schurter, P., Montané, A., and Tighe, R., "Structural analysis and modifications to the V. M. Blanco telescope for Dark Energy Camera (DECam) installation", Proc. SPIE 9145, 91455G (2014).

[7] Diehl, H. T., et al., "Dark Energy Survey Operations: Year 1", Proc. SPIE 9149, 91490V (2014).

[8] Lewis, P. M., Rogers, H., \& Schindler, R. H., "A radiometric all-sky infrared camera (RASICAM) for DES/CTIO", Proc. SPIE, 7735, 77353C (2010)

[9] Reil, K., Lewis, P., Schindler, R. H., \& Zhang, Z., "An update on the status and performance of the Radiometric All-Sky Infrared Camera (RASICAM)”, Proc. SPIE, 9149, 91490U (2014) 
[10] Blake, C. H., \& Shaw, M. M. 2011, PASP, 123, 1302.

[11]Li, T., DePoy, D. L., Kessler, R., et al., "aTmcam: a simple atmospheric transmission monitoring camera for sub $1 \%$ photometric precision”, Proc. SPIE, 8446, 84462L (2012)

[12] Li, T., DePoy, D. L., Marshall, J. L., et al., "Monitoring the atmospheric throughput at Cerro Tololo InterAmerican Observatory with aTmCam", Proc. SPIE, 9147, 91476Z (2014)

[13] Ruhl, J. E., et al., "The South Pole Telescope", Proc. SPIE 5498, 5498-11 (2004).

[14] Annis, J., et al., The SDSS Coadd: 275 degrees-squared of Deep SDSS Imaging on Stripe 82", arXiv:1111.6619.

[15] Kessler, R., Marriner, J., Childress, M., et al., "The Difference Imaging Pipeline for the Transient Search in the Dark Energy Survey", AJ 150, 172 (2015).

[16] https://cdevs.fnal.gov/redmine/projects/desops/wiki

[17] Rheault, J.-P., Depoy, D. L., Marshall, J. L., et al., "Spectrophotometric calibration system for DECam", Proc. SPIE 8446, 84466M (2012).

[18] Tucker, D. L., et al., in ASP Conf. Ser. Vol. 364, The Future of Photometric, Spectrophotometric and Polarimetric Standardization, Astron. Soc. Pac., ed. Sterken C., San Francisco, 187 (2007).

[19] Fitzpatrick, M. J., "DTS: The NOAO Data Transport System”, Proc. SPIE 7737, 77371T (2010).

[20] http://portal-nvo.noao.edu

[21] Pence, W. D., Seaman, R., \& White, R. L., "Lossless Astronomical Image Compression and the Effects of Noise", PASP 121, 414 (2009) and http://heasarc.nasa.gov/fitsio/fpack.

[22] Smith, R. C., Walker, A. R., and Miller, C., "DECam Community Pipeline Software requirements and technical Specifications", http://www.noao.edu/meetings/decam/media/community pipeline.pdf.

[23] Pence, W. D., White, R. L., \& Seaman, R., "Optimal Compression of Floating-point Astronomical Images without Significant Loss of Information”, PASP 122, 1065 (2010).

[24] Neilsen, E., and Annis, J., "OBSTAC: automated execution of Dark Energy Survey observing tactics", FERMILAB-CONF-13-397-CD.

[25] Mohr, J. J., et al., "The Dark Energy Survey Data Processing and Calibration System”, Proc. SPIE 8451, 84510D (2012).

[26] Zacharias, N., et al., Astronomical Journal 145, 44 (2013).

[27] Neilsen, E., Bernstein, G., Gruendl, R., and Kent, S., "Limiting magnitude, $\tau$, teff, and image quality in DES Year 1", FERMILAB-TM-2610-AE-CD (2015).

[28] Hendon, A. A., et al., JAAVSO 40, 430 (2012) and http://www.aavso.org/apass.

[29] http://www.ap-i.net/skychart/en/news/nomad.

[30] Goldstein, D. A., D’Andrea, C. B., Fischer, J. A., et al., “Automated Transient Identification in the Dark Energy Survey", AJ 150, 82 (2015).

[31] https://cdcvs.fnal.gov/redmine/projects/desops/wiki/Log of Configuration_Changes

[32] Roodman, A., "Focus and alignment of the Dark Energy Camera using out-of-focus stars", Proc. SPIE 8446, 844660 (2012).

[33] Roodman, A. J., Reil, K., Davis, C., "Wavefront sensing and the active optics system of the Dark Energy Camera", Proc. SPIE 9145, 914516 (2014).

[34] Soares-Santos, M., Kessler, R, Berger, E., et al., "A Dark Energy Camera Search for an Optical Counterpart to the First Advanced LIGO Gravitational Wave Event GW150914”, ApJL 823:L33 (2016).

[35] Annis, J., Soares-Santos, M., Berger, E., et al., "A Dark Energy Camera Search for Missing Supergiants in the LMC After the Advanced LIGO Gravitational Wave Event GW150914”, ApJL 823:L34 (2016).

[36] http://iri.columbia.edu/our-expertise/climate/forecasts/enso/current

[37] March, M., for the Dark Energy Survey Collaboration, "Initial supernovae results from the Dark Energy Survey”, Invited Talk at the APS March Meeting, Denver, CO, 2014.

[38] Davis, C., Rodriguez, J, and Roodman, A., "Wavefront-based PSF estimation", these proceedings.

[39] The smallest PSF DES exposure is 0.66" FWHM in z-band. For "Bragging Rights" see URL https://cdcvs.fnal.gov/redmine/projects/desops/wiki/Bragging rights (ie seeing records etc)

[40] Abbott, T. M. C., Walker, A. R., Points, S. D., et al., "The Blanco Telescope and its instruments: a status report", these proceedings.

[41] Gerdes, D. W., Jennings, R. J., Bernstein, G. M., et al., "Observation of Two New L4 Neptune Trojans in the Dark Energy Survey Supernova Fields”, AJ 15139 (2016). 
[42] Li, T. S., Balbinot, E., Mondrick, N, et al., "Discovery of a Stellar Overdensity in Eridanus-Phoenix in the Dark Energy Survey", accepted by ApJ.

[43] Bechtol, K., Drlica-Wagner, A., Balbinot, E., et al., "Eight New Milky Way Companions Discovered in FirstYear Dark Energy Survey Data", ApJ 807, 50 (2015).

[44] Drlica-Wagner, A., Bechtol, K., Rykoff, E. S., et al., "Eight Ultra-faint Galaxy Candidates Discovered in Year Two of the Dark Energy Survey", ApJ 813, 109 (2016).

[45] Crocce, M., Carretero, J., Bauer, A. H., et al., "Galaxy clustering, photometric redshifts and diagnosis of systematics in the DES Science Verification data", MNRAS 455, 4301 (2015)

[46]Zhang, Y., Miller, C., McKay, T., et al., "Galaxies in X-ray Selected Clusters and Groups in Dark Energy Survey Data I: Stellar Mass Growth of Bright Central Galaxies Since z 1.2”, ApJ 816, 92 (2016)

[47] Chang, C., Vikram, V., Jain, B., et al., "Wide-Field Lensing Mass Maps from DES Science Verification Data", Phys. Rev. Lett. 115, 051301 (2015)

[48] Gruen, D., Friedrich, O., Amara, A., et al., "Weak lensing by galaxy troughs in DES Science Verification Data", MNRAS 4553367 (2015)

[49] The Dark Energy Survey Collaboration, "Cosmology from Cosmic Shear with DES Science Verification Data", arXiv:1507.05552, submitted. 